\title{
Preoperative chemoradiation for locally advanced rectal cancer: comparison of three radiation dose and fractionation schedules
}

\author{
Shin-Hyung Park, MD, PhD, Jae-Chul Kim, MD, PhD \\ Department of Radiation Oncology, Kyungpook National University School of Medicine, Daegu, Korea
}

\begin{abstract}
Purpose: The standard radiation dose for patients with locally rectal cancer treated with preoperative chemoradiotherapy is 45-50 Gy in 25-28 fractions. We aimed to assess whether a difference exists within this dose fractionation range.

Materials and Methods: A retrospective analysis was performed to compare three dose fractionation schedules. Patients received 50 Gy in 25 fractions (group A), 50.4 Gy in 28 fractions (group B), or 45 Gy in 25 fractions (group C) to the whole pelvis, as well as concurrent 5 -fluorouracil. Radical resection was scheduled for 8 weeks after concurrent chemoradiotherapy.

Results: Between September 2010 and August 2013, 175 patients were treated with preoperative chemoradiotherapy at our institution. Among those patients, 154 were eligible for analysis $(55,50$, and 49 patients in groups $A, B$, and $C$, respectively). After the median follow-up period of 29 months (range, 5 to 48 months), no differences were found between the 3 groups regarding pathologic complete remission rate, tumor regression grade, treatment-related toxicity, 2-year locoregional recurrence-free survival, distant metastasis-free survival, disease-free survival, or overall survival. The circumferential resection margin width was a prognostic factor for 2-year locoregional recurrence-free survival, whereas ypN category was associated with distant metastasisfree survival, disease-free survival, and overall survival. High tumor regression grading score was correlated with 2-year distant metastasis-free survival and disease-free survival in univariate analysis.

Conclusion: Three different radiation dose fractionation schedules, within the dose range recommended by the National Comprehensive Cancer Network, had no impact on pathologic tumor regression and early clinical outcome for locally advanced rectal cancer.
\end{abstract}

Keywords: Rectal neoplasms, Neoadjuvant therapy, Chemoradiotherapy, Radiotherapy, Dose fractionation

\section{Introduction}

The mainstay of treatment for locally advanced rectal cancer (T3-4 and/or $\mathrm{N}+$ ) has been surgery. With the introduction of the total mesorectal excision method, a substantial decrease in the risk of locoregional failure has been achieved $[1,2]$. However, even with the total mesorectal excision method, surgery alone was found to have an inferior outcome compared to surgery combined with chemoradiotherapy (CRT) for locally advanced rectal cancer. The results of prospective randomized trials demonstrated that local control and survival were improved with adjuvant CRT $[3,4]$.

Although CRT administered both preoperatively and postoperatively could be beneficial, preoperative CRT became the standard treatment based on the German CAO/ARO/ AIO 94 trial, in which preoperative CRT was associated with

Received 14 March 2016, Revised 20 April 2016, Accepted 11 May 2016.

Correspondence: Jae-Chul Kim, MD, PhD, Department of Radiation Oncology, Kyungpook National University Hospital, 130 Dongdeok-ro, Jung-gu, Daegu 41944, Korea. Tel: +82-53-200-5352, Fax: +82-53-426-3303, E-mail: jckim@knu.ac.kr

(c) This is an Open Access article distributed under the terms of the Creative Commons Attribution Non-Commercial License (http://creativecommons.org/ licenses/by-nc/4.0/) which permits unrestricted non-commercial use, distribution, and reproduction in any medium, provided the original work is properly cited.

www.e-roj.org 
pathologic tumor response, improved tolerance, and increased likelihood of sphincter preservation in the distal rectum [5]. The Medical Research Council (MRC) CR07 trial, which reevaluated the role of preoperative radiotherapy (RT), showed that hypofractionated preoperative RT resulted in superior local control and disease-free survival (DFS) compared to selective postoperative RT [6].

The current National Comprehensive Cancer Network (NCCN) guidelines for rectal cancer recommend a total radiation dose of 45-50 Gy in 25-28 fractions for preoperative CRT. In our institution, three radiation dose fractionation schedules within this dose fractionation range were used for preoperative CRT in patients with locally advanced rectal cancer. We hypothesized that there is no difference in pathologic response and clinical outcome among the different radiation dose and fractionation schedules. The aim of this study was to evaluate pathologic response and clinical outcome among patients treated with 3 dose fractionation schedules.

\section{Materials and Methods}

We performed a retrospective analysis of patients with pathologically proven, locally advanced (cT3-4 and/or cN+) rectal cancer treated with preoperative CRT followed by radical resection at the institution. Between September 2010 and July 2013, 175 rectal cancer patients were treated with the aim of preoperative CRT. Of those patients, 12 developed distant metastasis during or after CRT, before radical resection (liver, 7; lung, 2; and distant lymph node, 3); 5 refused radical resection; and 4 did not complete the planned treatment schedule. Excluding those 21 patients, we reviewed 154 cases. Patient medical records were analyzed after approval was granted by the Kyungpook National University Hospital Institutional Review Board (IRB) with a waiver of the need for consent (IRB No. KNUH 2015-01-017). Data collected included patient factors (age, sex, and comorbidities), tumor factors (histology, clinical stage, pathologic stage, and completeness of resection), treatment factors (type of chemotherapy, dose of radiation therapy, type of surgery performed, and type of anastomosis), and clinical outcome (complications, tumor recurrence, and survival).

Pelvic radiotherapy was delivered with a four-field technique (anteroposterior/posteroanterior/right/left) using $10 \mathrm{MV}$ photon beams from a linear accelerator. Patients were placed in the prone position. The superior field edge was placed at the L5/ S1 interspace. The inferior field edge was at the anal verge or $2 \mathrm{~cm}$ below the tumor. Anteroposterior/posteroanterior fields had a 2-cm margin from the pelvic brim. Lateral treatment fields encompassed the entire sacrum posteriorly. The anterior border was 3 to $4 \mathrm{~cm}$ in front of the rectal wall. Three dose fractionation regimens were used: 56 patients were treated with 50 Gy in 2 Gy per fraction (group A), 50 were treated with $50.4 \mathrm{~Gy}$ in $1.8 \mathrm{~Gy}$ per fraction (group B), and 49 were treated with 45 Gy in 1.8 Gy per fraction (group C). Patients were not randomly allocated to these treatment groups, but dose fractionation regimens were selected based on their physician's preference and treatment policy at the time. Boost $\mathrm{RT}$ to primary tumor were not given. All fields were treated daily. Portal dosimetry was performed on the first day of treatment. Patients were examined weekly during the course of radiotherapy. In general, 2 cycles of 5 -fluorouracil (425 $\mathrm{mg} / \mathrm{m}^{2}$ for 4 consecutive days) and leucovorin $\left(20 \mathrm{mg} / \mathrm{m}^{2}\right.$ for 4 consecutive days) were administered intravenously during radiotherapy. A recovery period of 8 weeks after preoperative CRT was prescribed prior to surgery. During the recovery period, patients received one additional cycle of 5-fluorouracil/ leucovorin.

All patients were staged preoperatively and postoperatively according to the American Joint Committee for Cancer (AJCC) Staging Manual 7th edition. Pretreatment clinical staging was performed using abdominopelvic computed tomography, pelvic magnetic resonance imaging, and whole-body (upper legs to skull) ${ }^{18} \mathrm{~F}$-fluorodeoxyglucose positron emission tomography scanning.

Patients with no residual, viable tumor cells in the surgical specimen (ypTONO) were classified as having a pathologic complete response ( $\mathrm{pCR}$ ). To quantify tumor response to preoperative CRT, we used the standardized 5-point tumor regression grading (TRG) which was described by Dworak et al. [7]. The characteristics of each grade were as follows: grade 0 , no regression; grade 1 , minor regression (dominant tumor mass with obvious fibrosis in 25\% or less of the tumor mass); grade 2, moderate regression (dominant tumor mass with obvious fibrosis in 26\% to 50\% of the tumor mass); grade 3 , good regression (dominant fibrosis outgrowing the tumor mass; more than 50\% tumor regression); and grade 4, total regression (no viable tumor cells, only fibrotic mass). Positive circumferential resection margin (CRM) was defined as the presence of tumor cell within $1 \mathrm{~mm}$ or less from the inked radial margin.

Follow-up consisted of examinations every 3 months for the first year and then every 6 months subsequently. Most patients underwent pelvic computed tomography scans. Other imaging tests, such as pelvic magnetic resonance imaging and positron 
emission tomography scans, were performed, based on patient symptoms or signs. Follow-up data were obtained from patient medical records. Treatment-related toxicities were graded using the National Cancer Institute Common Terminology Criteria for Adverse Events (NCl-CTCAE) ver. 4.0 ( $\mathrm{NCl}$, Bethesda, MD, USA).

The primary endpoint of this study was pathologic tumor response after preoperative $C R T$; $p C R$ rate and TRG. The secondary endpoint included treatment-related complication, locoregional recurrence-free survival (LRFS), distant metastasis-free survival (DMFS), DFS and overall survival (OS). All events were measured from the date of the initiation of preoperative CRT. The time to the first defining event was assessed in terms of the following endpoints: LRFS, with recurrence at the primary site or regional lymph nodes as the event; DMFS, with disease metastasis to distant sites as the event; DFS, with any type of disease recurrence or death due to any cause as the event; and OS, with death due to any cause as the event. The LRFS, DMFS, DFS, and OS were estimated using the Kaplan-Meier method, and the differences were compared using log-rank tests. The $p$-values of $<0.05$ were considered statistically significant. Multivariate analysis using the Cox proportional hazards model was performed to identify independent predictors among the various potentia prognostic factors. Differences between patient characteristics were analyzed using chi-squared tests and one-way analysis of variance (ANOVA) when appropriate. Statistical analyses were performed using the $R$ statistical language ( $R$ Project ver 3.1.3) (http://www.R-project.org).

Table 1. Patient and treatment characteristics

\begin{tabular}{|c|c|c|c|c|}
\hline Characteristic & $\begin{array}{c}\text { Group A } \\
(50 \text { Gy in } 25 \mathrm{fx}) \\
(n=55)\end{array}$ & $\begin{array}{c}\text { Group B } \\
(50.4 \text { Gy in } 28 \mathrm{fx}) \\
(n=50)\end{array}$ & $\begin{array}{c}\text { Group C } \\
(45 \text { Gy in } 25 \mathrm{fx}) \\
(n=49)\end{array}$ & $p$-value \\
\hline Age $(y r)$ & $63(44-84)$ & $62(42-80)$ & $64(43-82)$ & 0.842 \\
\hline Gender (male:female) & $38: 17$ & $34: 16$ & $32: 17$ & 0.915 \\
\hline \multicolumn{5}{|l|}{ Location from anal verge $(\mathrm{cm})$} \\
\hline Mean \pm SD & $4.2 \pm 2.6$ & $4.3 \pm 2.4$ & $5.1 \pm 2.6$ & 0.072 \\
\hline$\leq 4$ & $32(58.2)$ & $25(50.0)$ & $21(42.9)$ & 0.576 \\
\hline $4-8$ & $19(34.5)$ & $19(38.0)$ & $21(42.9)$ & \\
\hline$\geq 8$ & 4 (7.3) & $6(12.0)$ & $7(14.3)$ & \\
\hline Pretreatment CEA (ng/mL) & & & & 0.930 \\
\hline$\leq 5$ & 39 (70.9) & $37(74.0)$ & $36(73.5)$ & \\
\hline$>5$ & $16(29.1)$ & $13(26.0)$ & $13(26.5)$ & \\
\hline \multicolumn{5}{|l|}{ Clinical staging } \\
\hline cT & & & & 0.746 \\
\hline 2 & $2(3.6)$ & $3(6.0)$ & $2(4.1)$ & \\
\hline 3 & $44(80.0)$ & $40(80.0)$ & $43(87.8)$ & \\
\hline 4 & $9(15.4)$ & $7(14.0)$ & 4 (8.2) & \\
\hline $\mathrm{cN}$ & & & & 0.943 \\
\hline 0 & $16(29.1)$ & $11(22.0)$ & $10(20.4)$ & \\
\hline 1 & $20(36.4)$ & $19(38.0)$ & $17(34.7)$ & \\
\hline 2 & $19(34.5)$ & $20(40.0)$ & $22(44.9)$ & \\
\hline Surgery type & & & & 0.589 \\
\hline LAR & $51(92.7)$ & $45(90.0)$ & 45 (91.8) & \\
\hline APR & $2(3.6)$ & $2(4.0)$ & $0(0)$ & \\
\hline ISR & $2(3.6)$ & $3(6.0)$ & $4(8.2)$ & \\
\hline Administration of adjuvant chemotherapy & & & & 0.964 \\
\hline Total & 46 (83.7) & $43(86.0)$ & 42 (85.7) & \\
\hline $5-\mathrm{FU}$ & $34(61.8)$ & $32(64.0)$ & $30(61.2)$ & \\
\hline Capecitabine & $9(16.4)$ & $10(20.0)$ & 9 (18.4) & \\
\hline FOLFOX & $3(5.5)$ & $1(2.0)$ & $3(6.1)$ & \\
\hline
\end{tabular}

Values are presented as median (range) or number (\%).

SD, standard deviation; CEA, carcinoembryonic antigen; LAR, low anterior resection; APR, abdominoperineal resection; ISR, intersphincteric resection; 5-FU, 5-fulorouracil; FOLFOX, folinic acid (leucovorin), fluorouracil, oxaliplatin. 
Table 2. Pathologic results

\begin{tabular}{|c|c|c|c|c|}
\hline & $\begin{array}{c}\text { Group A } \\
(50 \text { Gy in } 25 \mathrm{fx}) \\
(\mathrm{n}=55)\end{array}$ & $\begin{array}{c}\text { Group B } \\
(50.4 \text { Gy in } 28 \mathrm{fx}) \\
(\mathrm{n}=50)\end{array}$ & $\begin{array}{c}\text { Group C } \\
(45 \text { Gy in } 25 \mathrm{fx}) \\
(n=49)\end{array}$ & p-value \\
\hline Pathologic complete response & $12(21.8)$ & $13(26.0)$ & $10(20.4)$ & 0.786 \\
\hline Tumor regression grade & & & & 0.377 \\
\hline 0 & $0(0)$ & $3(6.0)$ & $2(4.1)$ & \\
\hline 1 & $10(18.2)$ & $9(18.0)$ & $10(20.4)$ & \\
\hline 2 & $16(29.1)$ & $9(18.0)$ & $6(12.2)$ & \\
\hline 3 & $17(30.9)$ & $16(32.0)$ & $21(42.9)$ & \\
\hline 4 & $12(21.8)$ & $13(26.0)$ & $10(20.4)$ & \\
\hline \multicolumn{5}{|l|}{ Pathologic staging } \\
\hline урТ & & & & 0.950 \\
\hline 0 & $12(21.8)$ & $13(26.0)$ & $10(20.4)$ & \\
\hline 1 & $4(7.3)$ & $2(4.0)$ & $1(2.0)$ & \\
\hline 2 & $11(20.0)$ & $10(20.0)$ & $11(22.4)$ & \\
\hline 3 & $27(49.1)$ & $24(48.0)$ & $25(51.0)$ & \\
\hline 4 & $1(1.8)$ & $1(2.0)$ & $2(4.1)$ & \\
\hline ypN & & & & 0.789 \\
\hline 0 & $42(76.4)$ & $42(84.0)$ & $41(83.7)$ & \\
\hline 1 & $10(18.2)$ & $6(12.0)$ & 7 (14.3) & \\
\hline 2 & $3(5.5)$ & $2(4.0)$ & $1(2.0)$ & \\
\hline Circumferential resection margin & & & & 0.868 \\
\hline Close $(\leq 1 \mathrm{~mm})$ & $2(3.6)$ & $3(6.0)$ & $4(8.2)$ & \\
\hline Negative (>1 mm) & $38(69.1)$ & $33(66.0)$ & $30(61.2)$ & \\
\hline Unknown & $15(27.3)$ & $14(28.0)$ & $15(30.6)$ & \\
\hline
\end{tabular}

Values are presented as number (\%).

\section{Results}

\section{Patient characteristics}

Patient characteristics were well balanced among the three dose-fractionation groups (Table 1). There were 104 male and 50 female. The median age was 63 years (range, 42 to 84 years). Clinical T category was T2, T3, and T4 in 7 (4.5\%), $127(82.5 \%)$, and $20(13.0 \%)$ patients, respectively. Clinical N category was N0, N1, and N2 in 37 (24.0\%), 56 (36.4\%), and 61 (39.6\%) patients, respectively. Tumor distance from the anal verge was $\leq 4,>4$ and $<8 \mathrm{~cm}$, or $\geq 8 \mathrm{~cm}$ in $78(50.6 \%)$, $59(38.0 \%)$, and $17(11.0 \%)$ patients, respectively. The median follow-up period was 29 months (range, 5 to 48 months).

\section{Acute toxicity of chemoradiotherapy}

All of the regimens were well tolerated. One hundred-six patients (68.8\%) experienced diarrhea. Grade 3 diarrhea developed in 10 patients, and no patients experienced grade 4 diarrhea. One patient experienced grade 2 radiation cystitis, which required conservative management. Grade 3 and 4 acute renal failure developed in 1 patient each after adjuvant chemotherapy, but neither patient showed progression to chronic renal failure. In summary, grade $\geq 3$ acute toxicity developed in 6 (10.9\%), 2 (4.0\%), and $3(6.1 \%)$ patients in groups $A, B$, and $C$, respectively, with no significant difference among the three groups $(p=0.368)$.

\section{Surgical procedures}

The median time from the end of preoperative CRT to radical resection was 58 days (range, 33 to 97 days). The type of surgery depended on the location of the tumor and the surgeon's decision. The most common operation performed was a low anterior resection in 141 patients (91.6\%). Other operations included abdominoperineal resection in 4 patients $(2.6 \%)$ and intersphincteric resection in 9 patients (5.8\%). The overall sphincter preservation rate was $97.4 \%$. There was no significant difference in the sphincter preservation rate among the three groups. Four patients in whom preservation of the sphincter failed had tumor extension to the anal verge. In a subgroup analysis of low rectal cancer $\leq 4 \mathrm{~cm}$ from anal verge, the sphincter preservation rate were $93.8 \%, 92 \%$, and $100 \%$ for group $A, B$, and $C$, respectively $(p=0.381)$. 
Table 3. Postoperative complications

\begin{tabular}{lccc}
\hline & $\begin{array}{c}\text { Group A } \\
(50 \text { Gy in 25 fx }) \\
(n=55)\end{array}$ & $\begin{array}{c}\text { Group B } \\
(50.4 \text { Gy in 28 fx }) \\
(\mathrm{n}=50)\end{array}$ & $\begin{array}{c}\text { Group C } \\
\text { (45 Gy in 25 fx }) \\
(\mathrm{n}=49)\end{array}$ \\
\hline Acute & & & \\
Wound infection & $1(1.8)$ & $2(4.0)$ & $0(0)$ \\
Anastomotic leak & $2(3.6)$ & $1(2.0)$ & $1(2.0)$ \\
Bowel obstruction & $0(0)$ & $1(2.0)$ & $0(0)$ \\
Bowel perforation & $1(1.8)$ & $0(0)$ & $0(0)$ \\
Fistula & $1(1.8)$ & $2(4.0)$ & $0(0)$ \\
Incisional hernia & $1(1.8)$ & $0(0)$ & $1(2.0)$ \\
Total & $6(10.9)$ & $6(12.0)$ & $2(4.1)$ \\
Chronic & & & $6(12.2)$ \\
Fecal frequency & $6(10.9)$ & $3(6.0)$ & $2(4.1)$ \\
Fecal incontinence & $2(3.6)$ & $0(0)$ & $0(0)$ \\
Impotence & $2(3.6)$ & $2(4.0)$ & $0(0)$ \\
Urinary incontinence & $2(3.6)$ & $0(0)$ & $8(16.3)$ \\
Total & $10(18.2)$ & $6(12.0)$ & 0.540 \\
\hline
\end{tabular}

Values are presented as number (\%).

\section{Pathologic results}

Pathologic results are summarized in Table 2. On examination of the resected specimens, pCR (TRG 4) was achieved in 35 patients $(22.7 \%)$. The $p C R$ rates in groups $A, B$, and $C$ were $21.8 \%, 26.0 \%$, and 20.4\%, respectively. Among 119 patients who failed to achieve $p C R$, TRG was grade 3 in 54 patients (35.1\%), grade 2 in 31 patients (20.1\%), grade 1 in 29 patients (18.8\%), and grade 0 in 5 patients (3.2\%). The good response rate (TRG $3+4$ ) was $57.8 \%$. No difference was found among the 3 dose-fractionation groups regarding the $p C R$ rate, TRG, or good response rate. Of the 119 patients who failed to achieve $\mathrm{pCR}$, CRM was measured in 110 patients. Nine patients (5.8\%) had a positive CRM (CRM $\leq 1 \mathrm{~mm}$ ). Among the three dose-fractionation groups, the incidence of positive CRM did not differ significantly. Pretreatment carcinoembryonic antibody level was not a predictor of pCR or TRG.

\section{Postoperative complications}

Table 3 shows the incidence of acute and chronic postoperative complications. The incidence of postoperative complications did not differ among the 3 dose-fractionation groups. Postoperative complications occurred in 37 patients (24.0\%), with 13 patients (8.4\%) experiencing acute complications and 24 patients (15.6\%) experiencing chronic complications. Acute postoperative complication rates were $10.9 \%, 12.0 \%$, and $4.1 \%$ in groups $A, B$, and $C$, respectively $(p=0.330)$. In the comparison between two groups (group $A+B$ vs. group $C$ ), acute postoperative complication rates were
$11.4 \%$ and $4.1 \%$ in group $A+B$ and group $C$, respectively ( $p$ $=0.140$ ). Anastomotic leakage was the most frequent acute postoperative complication and fecal frequency was the most frequent chronic complication. Three patients (1.9\%) died postoperatively, 1 from perianal abscess (group A), 1 from bowel perforation (group A), and 1 from bowel obstruction (group B). Grade 2 fecal incontinence developed in 4 patients (2.6\%). Grade 2 erectile dysfunction was recorded in 4 patients $(2.6 \%)$. Grade 2 urinary incontinence occurred in 2 patients $(1.3 \%)$.

\section{Locoregional recurrence-free survival (LRFS)}

Locoregional recurrence developed in 9 patients during the follow-up period. The 2-year LRFS for the entire patient population was $94.0 \%$. The 2-year LRFS rates were 98.0\%, $87.6 \%$, and $95.9 \%$ in groups $A, B$, and $C$, respectively ( $p=$ 0.293) (Table 4, Fig.1). Achievement of pCR (TRG 4) or good responsiveness according to TRG (TRG $3+4$ ) was not correlated with improvement in LRFS. A positive CRM was found to be correlated with worse LRFS in univariate $(p=0.034)$ but not in multivariate analyses (Table 4).

\section{Distant metastasis-free survival (DMFS)}

Distant metastasis occurred in 30 patients, and the 2-year DMFS rate for the entire patient population was $79.8 \%$. The 2-year DMFS rates in groups A, B, and C were $80.8 \%$, $84.5 \%$, and $74.0 \%$, respectively $(p=0.570$ ) (Table 4, Fig.1). The most frequent sites of distant metastasis were the lung 
and liver with $89.7 \%$ and $24.1 \%$, respectively. In univariate analysis, good responsiveness according to TRG ( $p=0.018)$, ypT category ( $p<0.001)$, ypN category $(p<0.001)$, and carcinoembryonic antigen level $(p=0.015)$ were associated with DMFS. In multivariate analysis, ypN category ( $p<0.001)$ showed a significant correlation with DMFS (hazard ratio $[H R]$, $3.94 ; 95 \%$ confidence interval [Cl], 1.79 to $8.65 ; p<0.001)$.

Table 4. Prognostic factors influencing actuarial rates of LRFS, DMFS, DFS, and OS

\begin{tabular}{|c|c|c|c|c|c|c|c|c|c|}
\hline \multirow{2}{*}{ Parameter } & \multirow{2}{*}{$\begin{array}{c}\text { No. of } \\
\text { patients }\end{array}$} & \multicolumn{2}{|c|}{ LRFS } & \multicolumn{2}{|c|}{ DMFS } & \multicolumn{2}{|c|}{ DFS } & \multicolumn{2}{|c|}{ OS } \\
\hline & & $2-y r(\%)$ & $p$-value & $2-y r(\%)$ & p-value & $2-y r(\%)$ & p-value & $2-y r(\%)$ & p-value \\
\hline Dose fractionation schedule of RT & & & 0.293 & & 0.570 & & 0.885 & & 0.117 \\
\hline A (50 Gy in $25 \mathrm{fx}$ ) & 55 & 98.0 & & 80.8 & & 77.3 & & 93.8 & \\
\hline B (50.4 Gy in $28 \mathrm{fx})$ & 50 & 87.6 & & 84.5 & & 78.0 & & 98.0 & \\
\hline C (45 Gy in $25 \mathrm{fx})$ & 49 & 95.9 & & 74.0 & & 72.4 & & 97.8 & \\
\hline Age (yr) & & & 0.941 & & 0.246 & & 0.201 & & 0.170 \\
\hline$\leq 60$ & 89 & 95.1 & & 77.3 & & 73.2 & & 95.1 & \\
\hline$>60$ & 65 & 92.6 & & 83.4 & & 80.0 & & 100 & \\
\hline Gender & & & 0.474 & & 0.419 & & 0.145 & & 0.325 \\
\hline Male & 104 & 94.5 & & 78.6 & & 73.0 & & 94.8 & \\
\hline Female & 50 & 95.9 & & 82.4 & & 82.7 & & 95.7 & \\
\hline Location from anal verge $(\mathrm{cm})$ & & & 0.659 & & 0.302 & & 0.383 & & 0.794 \\
\hline$<4$ & 57 & 93.5 & & 75.5 & & 71.9 & & 98.1 & \\
\hline$\geq 4$ & 97 & 94.2 & & 82.1 & & 78.3 & & 95.5 & \\
\hline Pretreatment CEA (ng/mL) & & & 0.621 & & 0.015 & & 0.045 & & 0.882 \\
\hline$\leq 5$ & 112 & 94.5 & & 84.8 & & 80.3 & & 96.2 & \\
\hline$>5$ & 42 & 92.9 & & 66.9 & & 65.2 & & 96.8 & \\
\hline cT category & & & 0.913 & & 0.327 & & 0.044 & & 0.084 \\
\hline $2-3$ & 134 & 95.0 & & 81.2 & & 78.8 & & 97.5 & \\
\hline 4 & 20 & 94.7 & & 70.3 & & 59.1 & & 89.4 & \\
\hline cN category & & & 0.450 & & 0.677 & & 0.787 & & 0.924 \\
\hline 0 & 37 & 92.8 & & 79.3 & & 76.9 & & 97.0 & \\
\hline $1-2$ & 117 & 94.3 & & 79.9 & & 75.8 & & 96.3 & \\
\hline Surgery type & & & 0.240 & & 0.556 & & 0.780 & & 0.271 \\
\hline LAR & 141 & 94.5 & & 80.5 & & 76.4 & & 96.1 & \\
\hline APR & 4 & 50.0 & & 100 & & 100 & & 100 & \\
\hline ISR & 9 & 100 & & 51.9 & & 51.9 & & 100 & \\
\hline Pathologic complete response & & & 0.919 & & 0.054 & & 0.021 & & 0.961 \\
\hline Yes & 35 & 97.0 & & 93.9 & & 93.9 & & 95.4 & \\
\hline No & 119 & 94.3 & & 75.6 & & 70.9 & & 100 & \\
\hline Tumor regression grade & & & 0.796 & & 0.018 & & 0.018 & & 0.367 \\
\hline $0-2$ & 65 & 92.7 & & 70.3 & & 66.8 & & 98.8 & \\
\hline $3-4$ & 89 & 94.9 & & 86.4 & & 82.8 & & 93.0 & \\
\hline ypT category & & & 0.716 & & $<0.001$ & & $<0.001$ & & 0.502 \\
\hline $1-2$ & 74 & 95.4 & & 94.0 & & 89.6 & & 98.6 & \\
\hline $3-4$ & 80 & 92.8 & & 66.3 & & 63.6 & & 94.3 & \\
\hline ypN category & & & 0.603 & & $<0.001$ & & $<0.001$ & & 0.031 \\
\hline 0 & 125 & 94.5 & & 81.6 & & 81.6 & & 98.3 & \\
\hline $1-2$ & 29 & 92.3 & & 53.2 & & 53.2 & & 87.8 & \\
\hline Circumferential resection margin $(\mathrm{mm})$ & & & 0.034 & & 0.373 & & 0.432 & & 0.312 \\
\hline$\leq 1$ & 9 & 59.3 & & 57.1 & & 50.8 & & 100 & \\
\hline$>1$ & 101 & 95.5 & & 77.5 & & 75.3 & & 96.6 & \\
\hline Unknown & 44 & 94.9 & & 87.6 & & 81.0 & & 95.4 & \\
\hline
\end{tabular}

LRFS, local recurrence-free survival; DMFS, distant metastasis-free survival; DFS, disease-free survival; OS, overall survival; RT, radiotherapy; CEA, carcinoembryonic antigen; LAR, low anterior resection; APR, abdominoperineal resection; ISR, intersphincteric resection. 
A

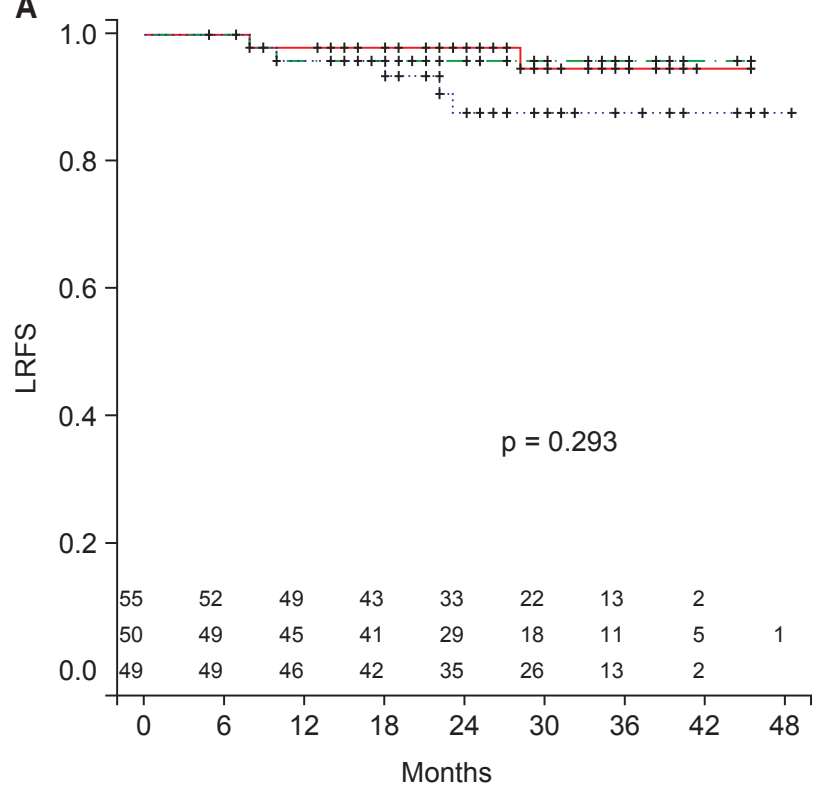

C

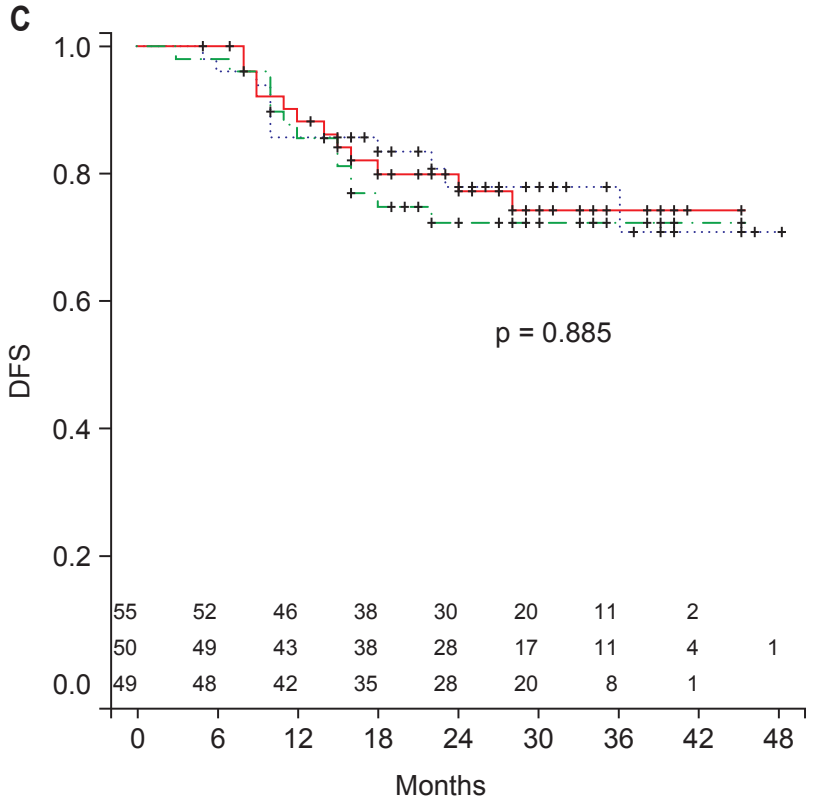

Group A (50 Gy in 25 fractions)

Group B (50.4 Gy in 28 fractions)

-.. Group C (45 Gy in 25 fractions)

B

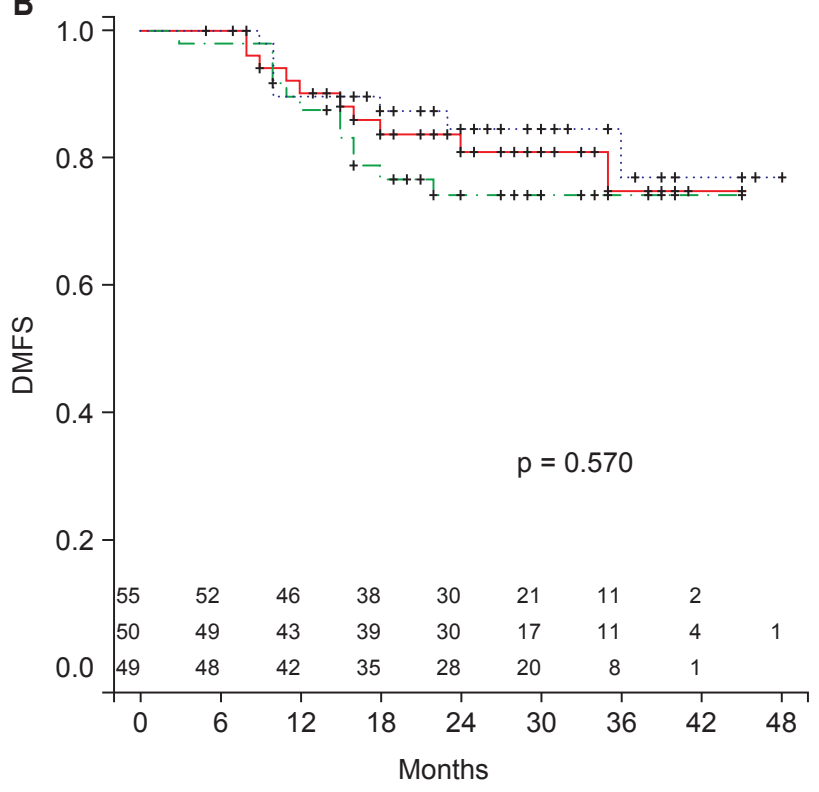

D

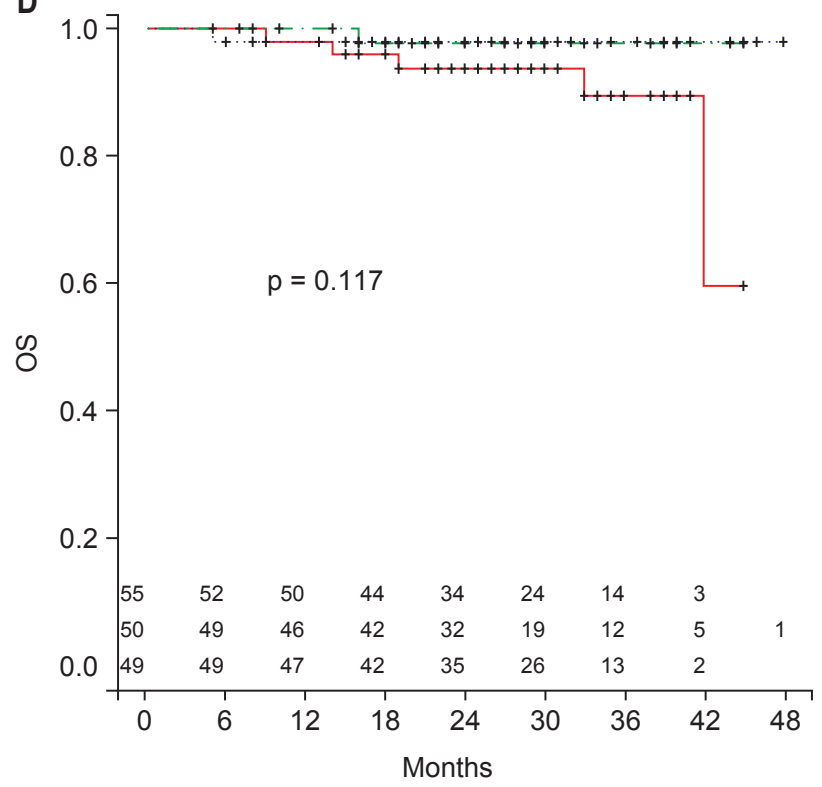

Fig 1. (A) Locoregional recurrence-free survival (LRFS), (B) distant metastasis free-survival (DMFS), (C) disease-free survival (DFS), and (D) overall survival (OS) according to three dose fractionation schedules.

\section{Disease-free survival (DFS) and overall survival (OS)} The 2-year DFS and OS rates for the entire patient population were $76.1 \%$ and $96.5 \%$, respectively. In groups $A, B$, and $C$, the 2-year DFS rates were $77.3 \%, 78.0 \%$, and $72.4 \%$, respectively $(p=0.885)$, and the 2-year OS rates were $93.8 \%, 98.0 \%$, and $97.8 \%$, respectively ( $p=0.117$ ) (Fig. 1). Achievement of $p C R$ ( $p$
$=0.021)$, good responsiveness of TRG $(p=0.018)$, ypT category ( $p<0.001)$, ypN category $(p<0.001)$, and carcinoembryonic antigen level $(p=0.045)$ were significantly correlated with DFS. In multivariate analysis, ypN category was associated with inferior DFS ( $H R, 3.40 ; 95 \% \mathrm{Cl}_{1} 1.63$ to $\left.7.09, \mathrm{p}=0.001\right)$. With respect to OS, ypN category was associated with OS in 
univariate $(p=0.031)$ and multivariate analyses $(H R, 6.27 ; 95 \%$ $\mathrm{Cl}, 1.21$ to $32.54 ; p=0.029)$.

\section{Discussion and Conclusion}

Although preoperative CRT has become the standard of treatment in patients with cT3-4 and/or N+ disease, there is still controversy as to the optimal radiation dose fractionation schedule. A long course of radiation therapy at a dose of 4550 Gy in 1.8-2 Gy per fraction over 5 to 5.5 weeks (as given in the German and National Surgical Adjuvant Breast and Bowel Project [NSABP] R-03 trials) has been thought to be "standard," whereas the short-course RT at a dose of $25 \mathrm{~Gy}$ in $5 \mathrm{~Gy}$ per fraction (as given in the MRC CR07 trial) is often used in parts of Europe. The Polish trial demonstrated that the short-course RT (25 Gy in 5 fractions) was as effective as the conventional fractionated long-course RT (50.4 Gy in 28 fractions) [8]. Though a higher pCR rate was seen with long-course RT (16\% vs. $1 \%$ ), along with fewer positive radial margins (4\% vs. 13\%) and considerable size reduction of the tumor, no differences in sphincter preservation rate, local control, or OS were observed. The Australian trial compared short-course (25 Gy in 5 fractions) and long-course CRT (50.4 Gy in 28 fractions) [9]. Despite the fact that pathologic tumor regression was significantly more common in patients randomly assigned to long-course RT, no difference in 3-year local control, 5-year OS, or late toxicity was found between the two arms of the trial. However, a meta-analysis of 21 randomized clinical trials showed that a biologically effective dose (BED) $>30 \mathrm{~Gy}_{10}$ with long-course RT showed a benefit in terms of the sphincter preservation rate, suggesting that the increased change in pathologic downstaging might lead to clinical benefits such as sphincter preservation [10]. Based on these results, longcourse RT has been recommended for sphincter preservation.

In a phase II dose escalation study by Chan et al. [11], 3 dose schedules (40 Gy/20 fractions, 40 Gy/20 fractions + postoperative $18 \mathrm{~Gy} / 9$ fractions, and $50 \mathrm{~Gy} / 25$ fractions) were compared, in which the schedule of $50 \mathrm{~Gy}$ was associated with improved 5 -year local control $(p=0.0011)$. Wiltshire et al. [12] compared 3 dose fractionation schedules (40 Gy in 20 fractions, $46 \mathrm{~Gy}$ in 23 fractions, and $50 \mathrm{~Gy}$ in 25 fractions). They reported that radiation doses $\geq 46$ Gy were associated with improvement in LRFS, DFS, and OS, but there was no difference between $46 \mathrm{~Gy}$ and $50 \mathrm{~Gy}$. The authors also showed a trend toward an increased $\mathrm{pCR}$ rate with higher doses $(15 \%, 23 \%$, and $33 \%$, respectively) ( $p=0.07)$. Gerard et al. [13] compared 2 dose fractionation schedules (45 Gy in 25 fractions and 50 Gy in 25 fractions) in a randomized fashion, although the chemotherapy regimens were different from each other (capecitabine and capecitabine + oxaliplatin, respectively). The $p C R$ rate was marginally improved $(p=0.09)$ and major pathologic response was improved significantly $(p=$ 0.02) with $50 \mathrm{~Gy}$ in 25 fractions. However, the interpretation of the results from the studies of Chen et al. [11] and Gerard et al. [13] needs to be cautious as they performed modifications to both radiation dose and chemotherapy regimens at the same time.

In contrast to previously mentioned studies [11-14], our study demonstrated no differences among the three dose fractionation schedules in terms of pathologic tumor response, locoregional recurrence, distant metastasis, and survival. Using the $\alpha / \beta$ ratio of $10 \mathrm{~Gy}$ for tumor cell kill, we calculated a BED10 of $60.0 \mathrm{~Gy}_{10}$ for group A (50 Gy in 25 fractions), of $59.5 \mathrm{~Gy}_{10}$ for group B (50.4 Gy in 28 fractions), and of 53.1 $\mathrm{Gy}_{10}$ for group C (45 Gy in 25 fractions). Thus, the radiation doses of groups $A$ and $B$ were similarly higher than those of group $C$. No statistically significant differences were found between group $A+B$ and group $C$. Thus, our study suggests that there might be no clear dose-response relationship within the BED range from $53.1 \mathrm{~Gy}_{10}$ to $60.0 \mathrm{~Gy}_{10}$. To examine the BED as a surrogate of normal tissue toxicity, BED3 was calculated using the $\alpha / \beta$ ratio of 3 . The BED3 of groups $A, B$, and $C$ were $83.3 \mathrm{~Gy}_{3}, 80.6 \mathrm{~Gy}_{3}$, and $72.0 \mathrm{~Gy}_{3}$, respectively. The acute postoperative complication rate was grossly higher in group $A+B\left(\geq 80.6 \mathrm{~Gy}_{3}\right)$ than in group C $(11.4 \%$ vs. $4.1 \%)$, although no statistical significance was found ( $p=0.140)$. It is possible that the dose-response relationship exists in terms of normal tissue toxicity because normal tissue generally has a smaller $\alpha /$ $\beta$ ratio than tumor cells within the BED range from $72.0 \mathrm{~Gy}_{3}$ to $83.3 \mathrm{~Gy}_{3}$.

In the present study, the achievement of pCR and good TRG score $(3+4)$ were associated with improved 2-year DMFS and DFS. Multiple studies have demonstrated the pathologic tumor response following preoperative CRT has a prognostic value for patients with locally advanced rectal cancer [15-17]. According to the recent updated results from the CAO/ARO/AI0-94 trial by Fokas et al. [18], complete (TRG 4) and intermediate TRG (TRG 2+3) were associated with improved DMFS and DFS after a median follow-up period of 132 months. Additionally, our results showed that higher TRG was associated with incremental CRM width in multivariate analysis. This suggests that greater the number of tumor cells killed by preoperative CRT, more adequate surgical margin would be achieved. Though the degree of pathologic tumor response such as $\mathrm{pCR}$ 
and TRG, have some prognostic value, radiation dose escalation for the achievement of them are need to be cautious.

Several phase III dose-escalation trials and a meta-analysis demonstrated a dose-response relationship with regard to $p C R$ for locally advanced rectal cancer [13,19-21]. Nevertheless, questions have existed as to the validity of pathologic tumor response for an early endpoint as a surrogate of long-term clinical outcome. In the analysis by Glynne-Jones et al. [22], pCR did not predict late outcomes such as local control or survival. The authors noted that whether we are actually altering the natural history of the disease with preoperative CRT or merely revealing a biological subgroup that would have had a favorable outcome anyway remained uncertain. Fokas et al. [18] also pointed out that the inherent aggressiveness of a tumor related to its biologic phenotype might reflect resistance to CRT and, at the same time, a higher incidence of distant metastasis. Appelt et al. [20] presented the results of a randomized phase III trial evaluating the addition of a brachytherapy boost (10 Gy in 2 fractions) to long-course preoperative CRT (50.4 Gy in 28 fractions) for locally advanced rectal cancer. They reported that, despite the fact that the degree of tumor response was higher in the brachytherapy boost arm, no differences in 5-year OS, progression-free survival, or LRFS were found. The authors noted that, although pathologic tumor regression was a prognostic factor, this did not imply that it was a valid surrogate clinical endpoint. In our study, as in that of Fokas et al. [18], TRG was associated with DMFS and DFS, but was not associated with LRFS. An association between pathologic tumor response and DMFS might suggest the possibility that CRT-resistant tumors which inherently had more biologic potential for distant metastasis were selectively included in the poor pathologic regression group. In this respect, it appears unreasonable to intensify the radiation dose for preoperative CRT based on results from the trials using pathologic tumor response as an endpoint.

The prognostic significance of the CRM status for local control and survival has been well established $[2,14,23,24]$. An analysis using data from the MRC CRO7 and National Cancer Institute of Canada Clinical Trials Group (NCIC-CTG) C016 trials found that the 3 -year local recurrence rates of CRMpositive patients and CRM-negative patients were 17\% and $6 \%$, respectively ( $p=0.0011$ ) [2]. Birbeck et al. [14] reported the clinical outcomes of 586 patients, of whom 165 patients had positive CRMs. CRM-positive patients showed significantly higher local recurrence rates (38\% vs. 10\%) and lower survival rates (40\% vs. 79\%) than CRM-negative patients. Similarly, in our study, a close CRM $(\leq 1 \mathrm{~mm})$ was associated with inferior 2-year LRFS (59.3\% vs. 95.5\%).

Our study has some limitations. First, there may be concealed selection biases due to its retrospective design, although patient and tumor characteristics seemed to be similar in each of the groups. Second, a small sample size might be insufficient to detect a potential statistical difference. Third, a relatively short follow-up period for oncologic outcomes is another limitation, especially for OS. Nevertheless, we think these results are still valid. Patients included in our retrospective study were treated during the same time period at a single institute; thus, RT and surgical techniques, pathologic evaluation, and chemotherapy were homogeneous.

In conclusion, comparison of three radiation dose fractionation schedules for locally advanced rectal cancer showed no impact of radiation dose fractionation schedules on the $\mathrm{pCR}$ rate and 2-year cumulative incidence of toxicity, LRFS, DMFS, DFS, or OS. Large prospective randomized trials are needed to confirm these results in the future.

\section{Conflict of Interest}

No potential conflict of interest relevant to this article was reported.

\section{References}

1. Arbman G, Nilsson E, Hallbook 0 , Sjodahl R. Local recurrence following total mesorectal excision for rectal cancer. Br J Surg 1996;83:375-9.

2. Quirke P, Steele R, Monson J, et al. Effect of the plane of surgery achieved on local recurrence in patients with operable rectal cancer: a prospective study using data from the $\mathrm{MRC}$ CR07 and NCIC-CTG C016 randomised clinical trial. Lancet 2009;373:821-8.

3. Kapiteijn E, Marijnen CA, Nagtegaal ID, et al. Preoperative radiotherapy combined with total mesorectal excision for resectable rectal cancer. N Engl J Med 2001;345:638-46.

4. van Gijn W, Marijnen CA, Nagtegaal ID, et al. Preoperative radiotherapy combined with total mesorectal excision for resectable rectal cancer: 12-year follow-up of the multicentre randomised controlled TME trial. Lancet Oncol 2011;12:575-82.

5. Sauer R, Becker $H_{1}$ Hohenberger $W$, et al. Preoperative versus postoperative chemoradiotherapy for rectal cancer. N Engl J Med 2004;351:1731-40

6. Sebag-Montefiore D, Stephens RJ, Steele R, et al. Preoperative radiotherapy versus selective postoperative chemoradiotherapy in patients with rectal cancer (MRC CR07 and NCIC-CTG C016): a multicentre, randomised trial. Lancet 2009;373:811-20. 
7. Dworak O, Keilholz L, Hoffmann A. Pathological features of rectal cancer after preoperative radiochemotherapy. Int J Colorectal Dis 1997;12:19-23.

8. Bujko K, Nowacki MP, Nasierowska-Guttmejer A, Michalski W, Bebenek M, Kryj M. Long-term results of a randomized trial comparing preoperative short-course radiotherapy with preoperative conventionally fractionated chemoradiation for rectal cancer. Br J Surg 2006;93:1215-23.

9. Ngan SY, Burmeister B, Fisher RJ, et al. Randomized trial of short-course radiotherapy versus long-course chemoradiation comparing rates of local recurrence in patients with $T 3$ rectal cancer: Trans-Tasman Radiation Oncology Group trial 01.04. J Clin Oncol 2012;30:3827-33.

10. Viani GA, Stefano EJ, Soares FV, Afonso SL. Evaluation of biologic effective dose and schedule of fractionation for preoperative radiotherapy for rectal cancer: meta-analyses and meta-regression. Int J Radiat Oncol Biol Phys 2011;80:985-91.

11. Chan AK, Wong AO, Langevin J, et al. Preoperative chemotherapy and pelvic radiation for tethered or fixed rectal cancer: a phase II dose escalation study. Int J Radiat Oncol Biol Phys 2000;48:843-56.

12. Wiltshire $K L$, Ward IG, Swallow $C$, et al. Preoperative radiation with concurrent chemotherapy for resectable rectal cancer: effect of dose escalation on pathologic complete response, local recurrence-free survival, disease-free survival, and overall survival. Int J Radiat Oncol Biol Phys 2006;64:709-16.

13. Gerard JP, Azria D, Gourgou-Bourgade S, et al. Comparison of two neoadjuvant chemoradiotherapy regimens for locally advanced rectal cancer: results of the phase III trial ACCORD 12/0405-Prodige 2. J Clin Oncol 2010;28:1638-44.

14. Birbeck KF, Macklin CP, Tiffin NJ, et al. Rates of circumferential resection margin involvement vary between surgeons and predict outcomes in rectal cancer surgery. Ann Surg 2002;235:449-57.

15. Hiotis SP, Weber SM, Cohen AM, et al. Assessing the predictive value of clinical complete response to neoadjuvant therapy for rectal cancer: an analysis of 488 patients. J Am Coll Surg
2002:194:131-5.

16. Valentini V, Coco C, Picciocchi A, et al. Does downstaging predict improved outcome after preoperative chemoradiation for extraperitoneal locally advanced rectal cancer? a longterm analysis of 165 patients. Int J Radiat Oncol Biol Phys 2002;53:664-74.

17. Capirci C, Valentini V, Cionini L, et al. Prognostic value of pathologic complete response after neoadjuvant therapy in locally advanced rectal cancer: long-term analysis of 566 ypCR patients. Int J Radiat Oncol Biol Phys 2008;72:99-107.

18. Fokas $E_{1}$ Liersch $T$, Fietkau $R$, et al. Tumor regression grading after preoperative chemoradiotherapy for locally advanced rectal carcinoma revisited: updated results of the CAO/ARO/ Al0-94 trial. J Clin Oncol 2014;32:1554-62.

19. Jakobsen A, Ploen J, Vuong T, Appelt A, Lindebjerg J, Rafaelsen SR. Dose-effect relationship in chemoradiotherapy for locally advanced rectal cancer: a randomized trial comparing two radiation doses. Int J Radiat Oncol Biol Phys 2012;84:949-54.

20. Appelt AL, Ploen J, Vogelius IR, Bentzen SM, Jakobsen A. Radiation dose-response model for locally advanced rectal cancer after preoperative chemoradiation therapy. Int J Radiat Oncol Biol Phys 2013;85:74-80.

21. Sanghera P, Wong DW, McConkey CC, Geh Jl, Hartley A. Chemoradiotherapy for rectal cancer: an updated analysis of factors affecting pathological response. Clin Oncol ( $R$ Coll Radiol) 2008;20:176-83.

22. Glynne-Jones R, Mawdsley S, Pearce T, Buyse M. Alternative clinical end points in rectal cancer: are we getting closer? Ann Oncol 2006;17:1239-48.

23. Nagtegaal ID, Marijnen CA, Kranenbarg EK, et al. Circumferential margin involvement is still an important predictor of local recurrence in rectal carcinoma: not one millimeter but two millimeters is the limit. Am J Surg Pathol 2002;26:350-7.

24. Wibe $A$, Rendedal PR, Svensson $E_{1}$ et al. Prognostic significance of the circumferential resection margin following total mesorectal excision for rectal cancer. Br J Surg 2002;89:327-34. 